ARTICLE

Received 9 Apr 2013 | Accepted 4 Mar 2014 | Published 7 Apr 2014

\title{
Phosphoregulation of Ire1 RNase splicing activity
}

\author{
Filippo Prischi ${ }^{1}$, Piotr R. Nowak ${ }^{1}$, Marta Carrara ${ }^{1} \&$ Maruf M.U. Ali
}

Ire1 is activated in response to accumulation of misfolded proteins within the endoplasmic reticulum as part of the unfolded protein response (UPR). It is a unique enzyme, possessing both kinase and RNase activity that is required for specific splicing of Xbp1 mRNA leading to UPR activation. How phosphorylation impacts on the Ire1 splicing activity is unclear. In this study, we isolate distinct phosphorylated species of Ire1 and assess their effects on RNase splicing both in vitro and in vivo. We find that phosphorylation within the kinase activation loop significantly increases RNase splicing in vitro. Correspondingly, mutants of Ire1 that cannot be phosphorylated on the activation loop show decreased specific Xbp1 and promiscuous RNase splicing activity relative to wild-type Ire1 in cells. These data couple the kinase phosphorylation reaction to the activation state of the RNase, suggesting that phosphorylation of the activation loop is an important step in Ire1-mediated UPR activation.

\footnotetext{
${ }^{1}$ Department of Life Sciences, Centre for Structural Biology, Sir Ernst Chain Building, Imperial College London, London SW7 2AZ, UK. Correspondence and requests for materials should be addressed to M.M.U.A. (email: maruf.ali@imperial.ac.uk).
} 
T he endoplasmic reticulum (ER) is the site of synthesis, modification and folding for membrane proteins and secretory proteins destined for other cellular compartments and the extracellular space. Upon ER stress, the influx of nascent polypeptides overwhelms the capacity of the ER to correctly fold and maintain protein tertiary structure, and, as a result, a complex cell signalling system termed the Unfolded Protein Response (UPR) is activated to maintain homeostasis. The UPR elicits a varied response, which includes transcriptional upregulation of chaperones, general cell translation attenuation, activation of ER-associated degradation (ERAD) and an increase in ER size. If the imbalance is not rectified, then the UPR switches from being prosurvival to instigating an apoptotic response ${ }^{1,2}$.

Ire1 is one of three sensor proteins that initiates and ultimately dictates the outcome of the response in humans. Ire1 consists of a luminal domain, a single pass transmembrane segment and a cytoplasmic domain that is further subdivided into an autophosphorylating kinase domain and an endoribonuclease (RNase) domain.

Upon activation, the luminal domains of Irel dimerize/ oligomerize; this positions the cytoplasmic kinase domains in close proximity to each other in a face-to-face orientation, which allows autophosphorylation to occur ${ }^{3}$. On the basis of yeast structures, Ire1 reorientates to form a back-to-back rearrangement, which may form higher oligomeric structures, and is thought to be important to achieve the RNase splicing competent state ${ }^{4,5}$.

In budding yeast, RNase domain splices a 252-nucleotide segment specific to HAC1 mRNA in a spliceosome-independent manner. The $3^{\prime}$ untranslated region of $\mathrm{HACl}$ contains a targeting sequence, which may be required for mRNA recruitment to the oligomerized Ire1 active state. By contrast, in the human system, unspliced $\mathrm{Xbp} 1$ contains a $\mathrm{C}$-terminal signal sequence that recruits the ribosome-nascent mRNA chain to the ER membrane to undergo splicing by Irel (refs 1,6). Irel splices a shorter 26-nucleotide sequence from Xbp1 mRNA. This causes a translational frameshift, which results in the expression of a potent transcriptional activator that upregulates expression of UPR target genes $2,7,8$.

UPR has been implicated in many diseased states, notably cancer. Interestingly, Ire1 has recently emerged as a new target for therapeutic intervention in multiple myeloma (MM), a cancer resulting from malignant transformation of plasma cells. Xbp1, besides its role in UPR, acts as a checkpoint control in plasma cell differentiation, and misregulation can cause uncontrollable proliferation leading to $\mathrm{MM}^{9-11}$. We have previously demonstrated that inhibiting the human Irel kinase reaction with a specific kinase inhibitor sunitinib resulted in loss of Xbp1 splicing in MM cell lines ${ }^{3}$.

Mechanistic understanding of how phosphorylation and ligand binding affect the RNase activity of Irel is of crucial importance for therapeutic interventions. Recently, phosphomimetic mutants of yeast Ire1 were seen to undergo sustained splicing, indicating that dephosphorylation of Ire1 is an important step in RNase deactivation ${ }^{12}$. However, a separate study suggested that the phosphoryl transfer reaction is important for deactivation rather than activation of $\mathrm{RNase}^{13}$, which supports a previous work suggesting that the kinase inhibitor 1NM-PP1 activated RNase splicing thereby circumventing the requirement for phosphorylation ${ }^{1,14}$. This contrasts with early experimental work showing mutation of catalytic residues in the kinase domain disrupting RNase function ${ }^{15}$ and more recently inhibition of kinase activity leading to loss of splicing in vivo 3,15,16. Furthermore, sunitinib has also been shown to be a potent inhibitor of closely related RNase L and PKR; these proteins are involved in antiviral innate immune responses and share significant sequence homology with Ire1 kinase ${ }^{17}$.

In an attempt to clarify the role of phosphorylation and ligand binding upon the RNase splicing activity of Ire1, we identify and map, using mass spectrometry analysis, specific phosphorylation sites by purifying distinct phosphorylated Ire1 populations from proteins expressed in the eukaryotic insect cell system. The isolation of differentially phosphorylated species allows us to assay the effects of specific phosphorylations upon the RNase splicing activity. We show that phosphorylations upon the activation loop increase the enzymatic rate of splicing manyfold above that of the dephosphorylated protein in vitro, whereas linker region and RNase domain phosphorylations have little/no impact upon splicing. Trans-autophosphorylation of dephosphorylated Irel in vitro leads to phosphorylation of two specific residues in the kinase activation loop. C-terminal Ire1 expressed in insect cells exhibits further phosphorylations that occur on the Linker and RNase domain. Furthermore, we show that mutations of activation loop phosphorylation sites result in loss of splicing and a reduction in RIDD activity relative to wild-type Ire1 in cells. This work indicates the importance of the kinase autotransphosphorylation reaction in activating the RNase domain to achieve the splicing active state, thereby coupling the two reactions together. Phosphorylation of kinase activation loop is an important and necessary step for achieving the activated Ire1 RNase splicing state in which splicing of Xbpl is enhanced, leading to subsequent activation of UPR signal. This study will help to clarify our understanding of Ire1-phosphoregulated RNase splicing and provide valuable insights for cancer therapeutic targeting of Ire1.

\section{Results}

Identification of phosphorylation sites. A cytoplasmic portion of human Ire1 (547-977) encompassing the kinase and endoribonuclease domains was expressed in insect cells. We have previously detailed our purification protocol for obtaining homogenous samples of dephosphorylated protein ${ }^{3}$. In the present protein purification, we omitted the incubation with lambda phosphatase and performed the monoQ anion exchange step with a very shallow gradient (Fig. 1a,b). From this purification step we were able to isolate several different peaks, which we sent for mass spectrometry analysis, where proteins were treated with trypsin digestion. The resultant peptide fragments were subjected to tandem mass spectrometry using MALDI-TOF/TOF and then ESI QTOF instruments (Supplementary Figs $1 \mathrm{~A}-\mathrm{D}$ and $2 \mathrm{~A}, \mathrm{~B}$ and Supplementary Table 1). Several peptides exhibited modifications consistent with being phosphorylated. Using the ESI QTOF setup, we were able to map the sites of certain phosphorylations. To further reinforce our data, we analysed the molecular mass of the different phosphorylated protein samples using the MALDI-TOF/TOF setup (Supplementary Fig. 3). Using both trypsin-digested analysis and molecular mass spectrometry of protein peak samples, coupled with the fact that this is an established technique for purifying phosphorylated proteins, particularly kinases ${ }^{18,19}$, we were fully confident of the phosphorylated protein assignments. The order of peaks that eluted off from the monoQ column roughly correlated with increasing number of phosphorylations upon Irel. The first peak (Fig. 1a,b), which was also the largest peak, relates to purified Ire1 protein being in a dephoshorylated state, interestingly, suggesting that Irel protein is being kept in a 'ground dephosphorylated state' by various phosphatases consistent with other kinase regulatory mechanisms. The next peak (PK2) (Fig. 1a,b) indicated purified protein being phosphorylated at two sites ser551 and 


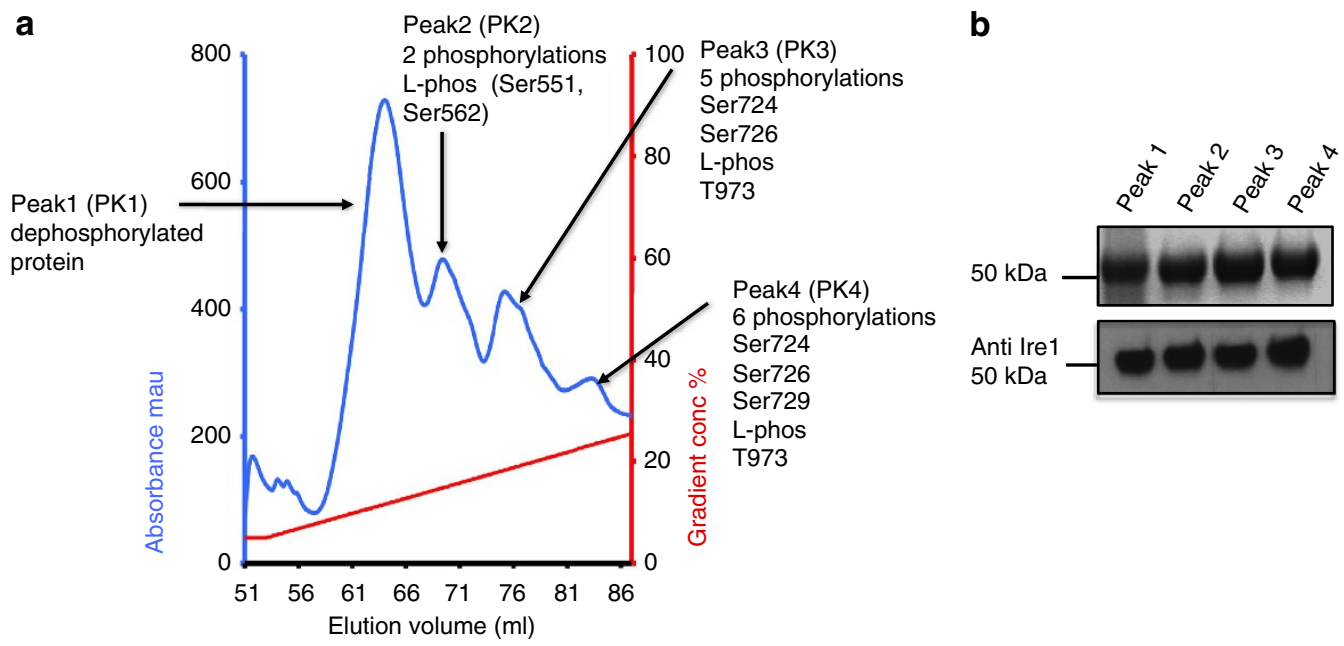

b

C
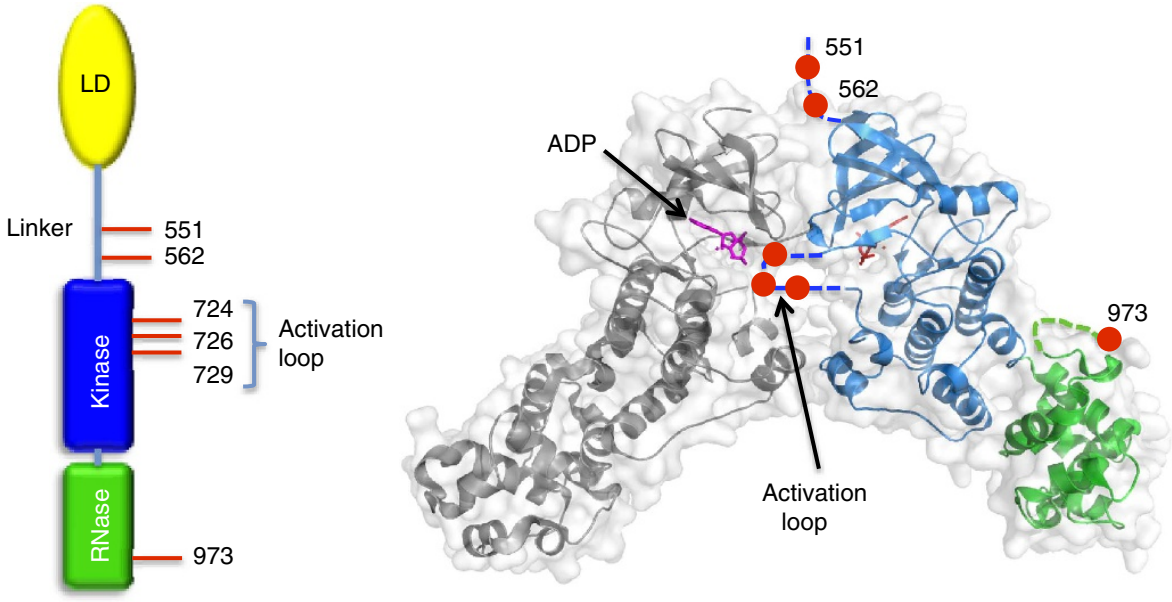

Figure 1 | Identification of phosphorylation sites. Chromatogram showing the elution profile of human Ire1 protein (547-977) expressed in insect cells and passed through a monoQ anion exchange column. The four peaks indicated distinct phosphorylated species of Ire1, and their sites of phosphorylation were identified by mass spectrometry (see Supplementary Figs 1,2, Supplementary Table 1). (b) Ire1 protein (547-977) samples from the four distinct peaks isolated by anion exchange were visualized by SDS-PAGE (top panel) and by western blot using a generic human Ire1 antibody (bottom panel). (c) The position of phospho sites relative to each other and mapped onto the X-ray structure of human Ire1 autophosphorylation complex (3P23).

ser562 in the linker region; we refer to this as L-phos. Peak 3 (PK3) contained five phosphorylations at ser724, ser726, and L-phos, with a weak/low signal for thr973 phosphorylation. PK4 indicates Ire1 protein phosphorylated at ser724, ser726, ser729 and L-phos, and, again as with PK3, weak/low levels of thr973 phosphorylation were detected. The sites of phosphorylations fall into three particular areas, phosphorylation in the linker region (L-phos), kinase activation loop phosphorylations and thr973 phosphorylation positioned within the C-terminal RNase domain (Fig. 1c). The isolation of differentially phosphorylated Ire1 subspecies allows us to assay the effects of specific phosphorylations upon Ire1 RNA splicing activity; however, initially it is also necessary to understand the effects of ligand binding upon the splicing reaction.

Ligand binding has minimal effect on RNase activity in vitro. To assay the effects of phosphorylations and ligand binding upon RNase splicing activity, we made use of fluorescence resonance energy transfer (FRET) - quenched mini Xbpl RNA substrate probe (Fig. 2a), which when cleaved by Irel emits fluorescence at $590 \mathrm{~nm}$ (cy3) wavelength ${ }^{20}$. Initially, just taking the dephosphorylated protein, we incubated varying concentrations of substrate probe and measured the fluorescence emitted over a period of time (Fig. 2b). The resultant reaction data were of very high quality and the reactions fitted excellently with MichaelisMenton enzyme kinetics. By using the initial rates of each reaction at varying concentrations of the probe, we were able to successfully measure the substrate turnover $\left(K_{\text {cat }}\right)$ and the catalytic enzyme efficiency $\left(K_{\text {cat }} / K_{\mathrm{m}}\right)$ (Table 1$)$. We performed the same analysis for both dephosphorylated proteins in the presence of a non-hydrolysable ATP mimic AMP-PNP and ADP (Fig. 2c). We found that the $K_{\text {cat }}$ values for both the dephosphorylated and the AMP-PNP bound cofactors were almost identical at 0.118 and $0.144 \mathrm{~s}^{-1}$; this was also reflected in the $k_{\mathrm{cat}} / K_{\mathrm{m}}$, which gives a measure of the efficiency of the enzyme, at $3.21 \times 10^{4}$ and $2.63 \times 10^{4} \mathrm{M}^{-1} \mathrm{~s}^{-1}$, respectively. For dephoshorylated protein in the presence of ADP, the $K_{\text {cat }}$ and $K_{\text {cat }} / K_{\mathrm{m}}$ were only slightly higher at $0.295 \mathrm{~s}^{-1}$ and $4.61 \times 10^{4}$ $\mathrm{M}^{-1} \mathrm{~S}^{-1}$, suggesting that the bound ADP may assist in a conformation conducive for the RNase cleavage reaction. Previously reported data in the field suggested that the presence of nucleotide can induce RNase activity; if this were the case, then AMP-PNP would also have an inducing effect, which in our data it does not and is instead clearly identical to the basal level splicing of dephosphorylated protein without any ligand, 
a

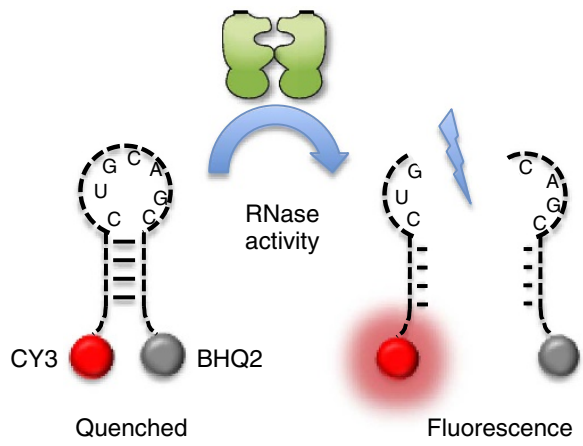

b

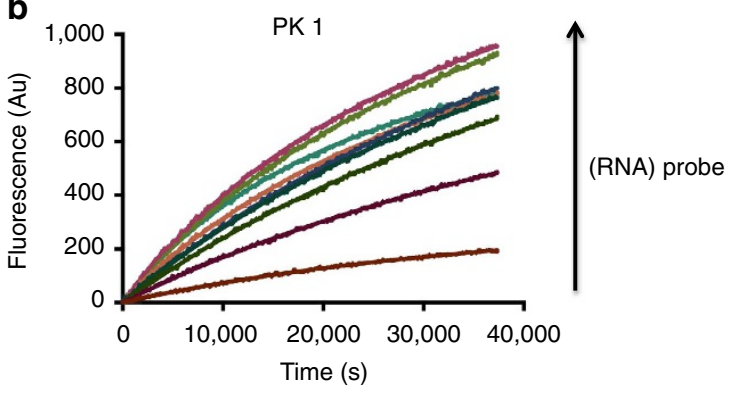

c

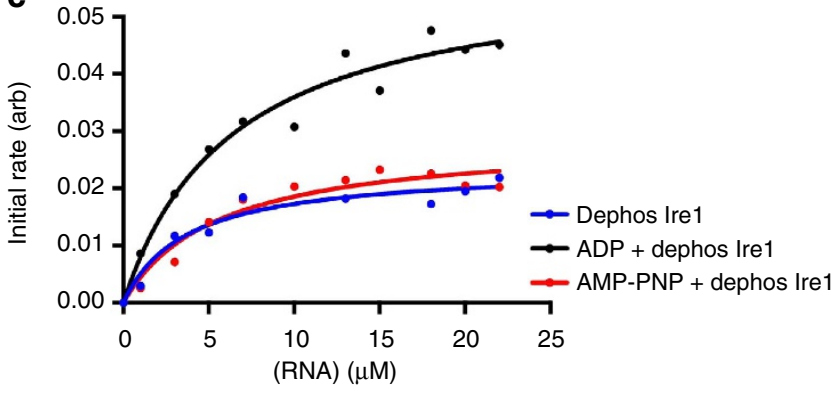

Figure 2 | Ligand binding to dephosphorylated Ire1 has little impact on RNase splicing. (a) Fret-quenched RNA Xbp1 substrate probe was tagged with Cy3 label at the $5^{\prime}$ end and with BHQ2 quencher at $3^{\prime}$ end. The substrate probe was cleaved by Ire1 allowing measurement of fluorescence emission at $590 \mathrm{~nm}$. (b) Fluorescence emission time course experiment measuring RNase splicing of FRET probe by Ire1 in vitro. Dephosphorylated Ire1 protein was incubated with substrate probe, and cleavage reaction was followed by fluorescence emission over a period of time. The time course was repeated with varying concentrations of the substrate probe. All experiments were performed in triplicate. (c) The initial rates of reactions calculated from each profile obtained from fluorescence time course splicing experiment in $\mathbf{b}$ were plotted against various concentrations of substrate probe to give initial rate curves that obeyed Michelis-Menton kinetics. The initial rate curves for both dephosphorylated Ire1 (blue) and dephosphorylated Ire1 bound to AMP-PNP (red) gave similar profiles, whereas dephosphorylated Ire1 bound to ADP (black) gave a slightly elevated profile (see Table 1).

suggesting that just the mere presence of nucleotide is not enough to induce significant splicing. However, on comparison of the dephosphorylated Ire1 rates of reaction with those of the phosphorylated Ire1 protein (see below PK3 and PK4), the values between dephosphorylated, ADP and AMP-PNP bound protein showed insignificant differences.

Activation loop phosphorylation increases RNase activity. The purification and isolation of the differentially phosphorylated Ire1 enabled us to measure the effects of specific phosphorylations upon the RNase activity (Fig. 3a-e). PK2 contains doubly phosphorylated protein in the linker region at ser551 and ser562. Analysis of the initial rate curve for PK2-phosphorylated Ire1 (Fig. 3a,e and Table 1) gave a $K_{\text {cat }} / K_{\mathrm{m}}$ value of $5.11 \times 10^{4} \mathrm{M}^{-1}$ $\mathrm{s}^{-1}$. This value is only slightly higher than that of dephosphorylated Irel protein with ADP binding, suggesting that phosphorylations within the linker region do not have much of an effect on the catalytic efficiency of Irel splicing reactions, which is not that surprising as it is quite a distance away from the RNase and indeed kinase active sites.

PK3 contains Ire1 protein, which is phosphorylated on L-phos, and three further sites, ser724 and ser726 on the activation loop of the kinase domain and low levels of pthr973 in the RNase domain. Using the FRET assay, we obtained a $K_{\text {cat }}$ value of $7.37 \mathrm{~s}^{-1}$ and $K_{\text {cat }} / K_{\mathrm{m}}$ of $3.02 \times 10^{5} \mathrm{M}^{-1} \mathrm{~s}^{-1}$ (Fig. $3 \mathrm{~b}, \mathrm{e}$ and Table 1). These values represent a huge increase over the dephosphorylated protein or indeed any of the previous samples tested. The substrate turnover $\left(K_{\text {cat }}\right)$ was over 60 -fold higher than that for the dephosphorylated protein and at least 24 -fold higher than that for dephosphorylated Irel with ADP bound. These increases were also mirrored in the $K_{\text {cat }} / K_{\mathrm{m}}$ values with PK3 Ire1 being an order of magnitude higher than that of dephosphorylated protein. The difference between PK2 and PK3 is essentially of the two phosphorylations, ser724, ser726 (with low level thr973 detected), on the activation loop. These two phosphorylations are responsible for the greatest increase in enzyme RNase activity, even more so than that of PK3 to PK4 (see below PK4). The phosphorylations no doubt have an effect on the conformation of the activation loop and possibly lock it into a position different from that of the dephosphorylated protein. These movements would transmit down to the RNase domain thus allowing it to adopt a conformation, which is more conducive for splicing to occur.

PK4 Ire1 differs from PK3 protein by having one additional phosphorylation positioned on the activation loop at ser729. Using the FRET assay, we obtained the $K_{\text {cat }}$ value of $12.7 \mathrm{~s}^{-1}$ and $K_{\text {cat }} / K_{\mathrm{m}}$ value of $1.26 \times 10^{6} \mathrm{M}^{-1} \mathrm{~s}^{-1}$ (Fig. $3 \mathrm{c}$,e and Table 1 ). This represents an increase of more than 105-fold in substrate turnover compared with dephoshorylated protein and almost double that of PK3 Ire1. Again, this is reflected in the catalytic efficiency of $K_{\text {cat }} / K_{\mathrm{m}}$, which is almost 40 -fold higher than that of dephosphorylated protein and over 4-fold higher than that of PK3 Ire1. Although the enzymatic rate values for PK4 are the highest for all samples tested, the greatest increase between samples is that of PK2 to PK3 and is attributable to pS274 and pS726.

It is clear from the enzymatic rate analysis that the phosphorylations on the activation loop enhance the RNase splicing activity of Ire1 manyfold above that of the dephosphorylated protein; in the case where all three of the activation loop residues, ser724, ser726 and ser729, are phosphorylated, the substrate turnover is over 100 -fold higher than that of the dephosphorylated protein. This unambiguously links the phosphorylations upon the activation loop to enhanced RNase splicing of Irel. The phosphorylations upon the linker region and the RNase domain do not seem to effect the splicing reaction. Similarly, the addition of nucleotide ADP and AMP-PNP to dephosphorylated protein seems to have a minimal effect upon the splicing reaction when compared with activation loop phosphorylations. It would be interesting to see how the binding of ligands to the distinct phosphorylated species effects the RNase activity. To test this point, we incubated both ADP and AMPPNP to the different Irel peaks and observed the enzymatic activity (Supplementary Fig. 4A-C). We see that, in the presence of ADP and AMP-PMP, the enzymatic reaction is largely reduced when compared with that of phosphorylated protein without 
Table 1 | Enzymatic rates of RNase splicing; the kinetic parameters of various dephosphorylated and phosphorylated states of Ire1.

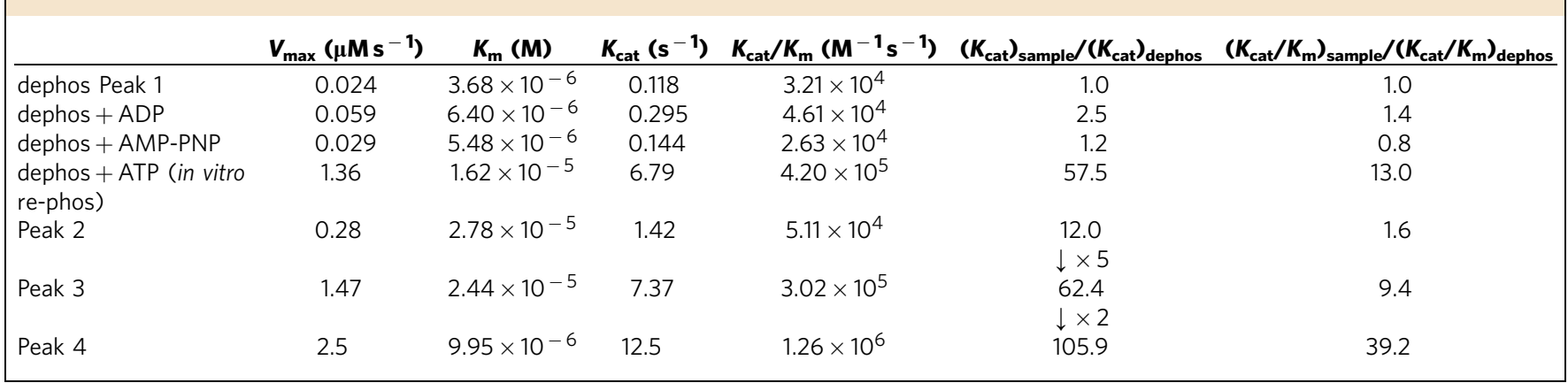

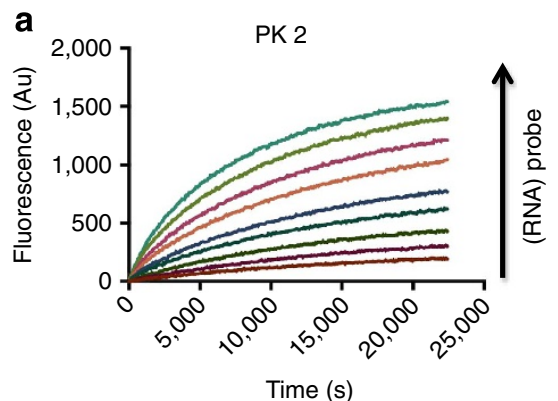

d

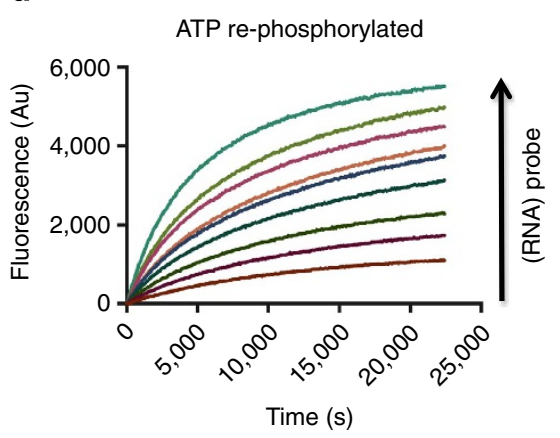

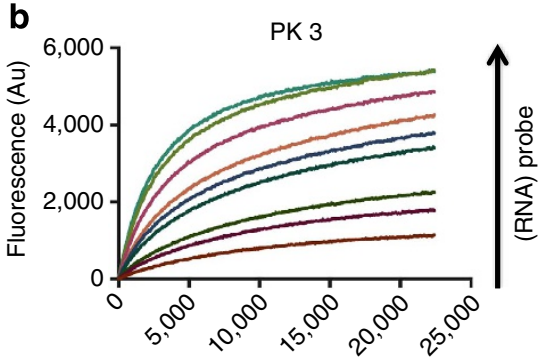

Time (s)
C

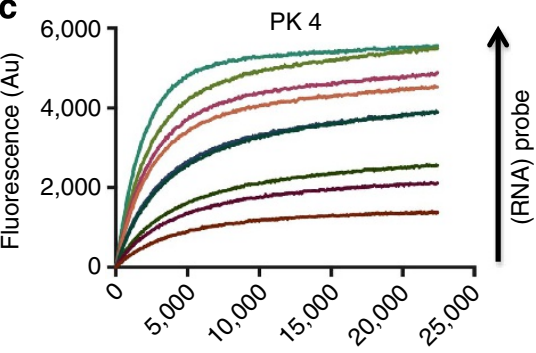

Time (s)

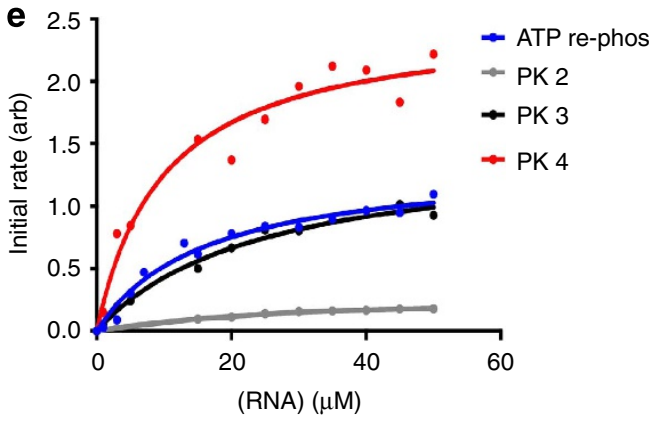

Figure 3 | Distinct phosphorylations significantly increase RNase splicing. (a-d) Fluorescence time course experiments measuring splicing of FRET probe by differently phosphorylated species of Ire1 protein at increasing concentrations of substrate probe. (a) Profile of PK 2 Ire1 protein. (b) Profile of in vitro ATP re-phosphorylated Ire1 that was initially dephosphorylated. (c) Profile of PK 3 Ire1 protein. (d) Profile of PK 4 Ire1 protein. (e) A plot of the initial rates derived from each fluorescence time course splicing profile in a-d at varying substrate concentrations to give an overall initial rate curve for that particular protein peak sample from which we obtain the kinetic parameters listed in Table 1. PK 4 Ire1 sample (red) displays the steepest initial rate curve profile, and this is reflected in its kinetic rates $K_{\mathrm{m}}$ and $K_{\text {cat }}$ (see Table 1). PK 3 human Ire1 protein sample (black) purified from insect cells with distinct phosphorylations on ser724 and ser726 within the activation loop and with extra L-phos and pT973 gives a very similar initial rate profile to dephosphoshorylated protein that has been re-phosphorylated by incubating with ATP in vitro and exhibiting phosphorylations on Ser724 and Ser726 on activation loop only. PK 2 Ire1 protein sample (grey) containing only L-phos modification shows a very slow initial rate curve profile.

ligand. This indicates that ADP and AMP-PMP, rather than having an activating effect upon splicing, reduce the rate of reaction significantly. Ligand binding possibly causes the glycinerich loop to close over the ligand-bound active site, locking the kinase domain in a 'closed' conformation, with a subsequent conformational change transmitted to RNase domain and negatively effecting the splicing activity.

In vitro Ire1 is re-phosphorylated on ser724 and ser726. The distinct phosphorylated populations of Irel purified from insect cells, which contain phosphatases, indicate that they are biologically relevant. It would be interesting to investigate which of these phosphorylations occur solely due to Ire1 kinase autotransphosphorylation reaction. To test this point, we incubated dephosphorylated protein in the presence of ATP and then subjected the protein to trypsin digestion and subsequent mass spectrometry analysis. The mass spectrometry analysis indicated the presence of two phosphorylations at ser724 and ser726 on the activation loop of the kinase domain. We then tested the substrate turnover and catalytic efficiency by using the FRET probe assay (Fig. 3d,e and Table 1). The $K_{\text {cat }}$ and $K_{\text {cat }} / K_{\mathrm{m}}$ values for the in vitro re-phosphorylated Ire1 wwere $6.79 \mathrm{~s}^{-1}$ and $4.2 \times$ $10^{5} \mathrm{M}^{-1} \mathrm{~s}^{-1}$, respectively. These values match very closely with 
those of PK3 Ire1, which was also phosphorylated on ser724 and ser726 of the activation loop with further phosphorylations on L-phos and Thr973, but as L-phos and pThr973 do not contribute significantly to the splicing reaction, we would expect that PK3 and re-phosphorylated protein to have similar values for their enzymatic rates, which is indeed the case. This further supports the notion that L-phos and pThr973 do not contribute greatly to splicing reaction kinetics, and the major factor in the increase in RNase activity is due to phosphorylation of activation loop residues ser724, ser726 and ser729 with the greatest contribution from ser724 and ser726.

Activation loop mutant reduces RNase splicing in cells. It is clear that activation loop phosphorylations have a significant effect upon the RNase activity in vitro. We wanted to investigate the effects of mutating specific phosphorylations on RNase splicing in vivo. To address this point, we co-transfected HT1080 cells with plasmids expressing Ire1 phosphorylation mutations (Fig. 4a-c, Supplementary Fig. 5), identified from our mass spectrometry data, and measured splicing by RT-PCR analysis. Initially, we mutated each phosphorylation site to assay the effect of that specific phosphorylation upon splicing and then used the double and triple mutations to mimic sets of phosphorylations such as L-phos (S551A, S562A), in vitro re-phosphorylated activation loop (S724A, S726A) and full activation loop phosphorylations (S724A, S726A, S729A) to establish their effects. Using $50 \mu \mathrm{M}$ tunicamycin to induce ER stress, we measured the total level of splicing for each mutant and compared it with both wild-type Irel and empty vector control. After $2 \mathrm{~h}$, the most drastic effect by a single-amino-acid mutation on splicing was observed with activation loop mutants, S724A, S726A, S729A and, in particular, S726A (Fig. 4a-c), which displayed a $50 \%$ reduction in splicing when compared to wild type. This is in agreement with the in vitro splicing activity where the biggest effect was seen with PK3, which contains both S724 and S726. The linker region mutations S551A and S562A seem to have very little effect upon splicing; this is further confirmed by the double linker region mutation S551A/S562A, which also did not exhibit significant loss of splicing compared with activation loop mutations. The T973A mutation displayed a slight inhibition of splicing of around $85 \%$ of wild type, which was more than L-phos double mutant, but again when compared with the activation loop mutants it becomes less significant. The phosphorylation on residues pS724 and pS726, which is present in PK3 and in in vitro re-phosphorylated Ire1, was seen from our FRET assay to have the largest increase in activity (Table 1) corresponding to a fivefold jump from PK2 to PK3, whereas PK3 to PK4 represented a twofold increase in terms of $K_{\text {cat }}$ values. It is therefore straightforward to predict that the double-mutant S724A/S726A would have a drastic effect and indeed this was the case with splicing in vivo reduced to $38 \%$, recapitulating the results obtained in vitro. Interestingly, the triple activation loop mutant S724A, S726A, S729A seems to be inhibiting to a similar level as that of the double-mutant S724A, S726A. This indicates that there maybe a basal level of splicing, which is not directly influenced by phosphorylation, and that mutation of S724A and S726A is sufficient to reach this basal level in vivo.

Therefore, these data clearly indicate that mutation of activation loop phosphorylation sites upon Ire1 in HT1080 cells significantly reduces RNase splicing, consistent with the in vitro FRET splicing data.

Activation loop mutant reduces splicing in Ire1 - / - cells. To further emphasize the importance of the activation loop phosphorylations, we conducted a splicing time course
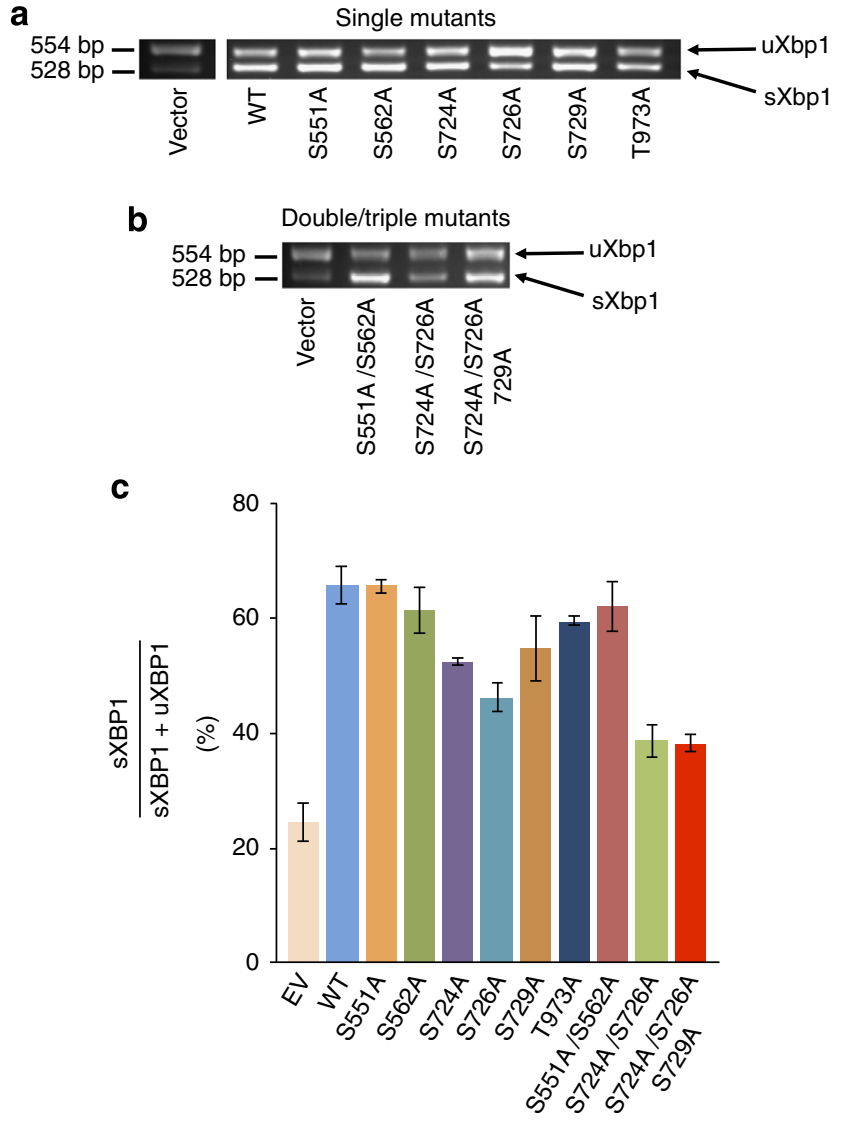

Figure 4 | Mutation of phosphorylation sites inhibits splicing in cells. (a) Xbp1 splicing in HT 1080 human cell line. Individual phosphorylation mutants were co-transfected into HT1080 cells and subjected to tunicamycin for $2 \mathrm{~h}$ to induce ER stress. RT-PCR of sXbp1 and uXbp1 was run on 3\% agarose gel and visualized by gel red DNA stain. Empty vector was used as control, and all experiments were repeated in triplicate. (b) Xbp1 splicing using sets of mutations mimicking groups of phosphorylations within Ire1. S551A/S562A represents L-phos mutations, S724A/S726A replicates in vitro re-phosphorylation of dephos Ire1 with ATP, S724A/S726A/S729A triple mutant represents all activation loop phosphorylations. (c) Quantification of inhibition of Xbp1 splicing in vivo of each individual phosphorylation mutant and sets of mutants in HT1080 cells, with empty vector (EV) as control. Splicing is clearly reduced in all activation loop mutants and especially in the double and triple mutants recapitulating the in vitro findings. Linker region or L-phos single and double mutants seem not to have an impact on splicing, whereas T973A seems to have a small impact. Error bars represent mean \pm s.e.m., $n=3$.

experiment using Ire1 - / - MEF cells in mild ER stress conditions of $0.5 \mu \mathrm{M}$ tunicamycin with triple activation loop mutant S724A, S726A, S729A and wild-type Ire1 and observed the fold change in splicing compared with empty vector by qRT-PCR analysis (Fig. 5a). We see at each time point a reduced level of splicing for the triple activation loop mutant as compared with wild type. At $2 \mathrm{~h}$, the mutant level of splicing is comparable to empty vector splicing but increases twofold above empty vector before dropping back after $18 \mathrm{~h}$. The wild type displays significantly more splicing at each time point with an almost 3.5-fold increase at $6 \mathrm{~h}$ before dropping back at $18 \mathrm{~h}$. Moreover, the splicing for wild type fluctuates more than the mutant. This result clearly shows that mutation of active site phosphorylations reduces splicing in mild ER stress conditions in Ire $1-/-$ cells and is consistent with both the HT1080 cell line and in vitro 


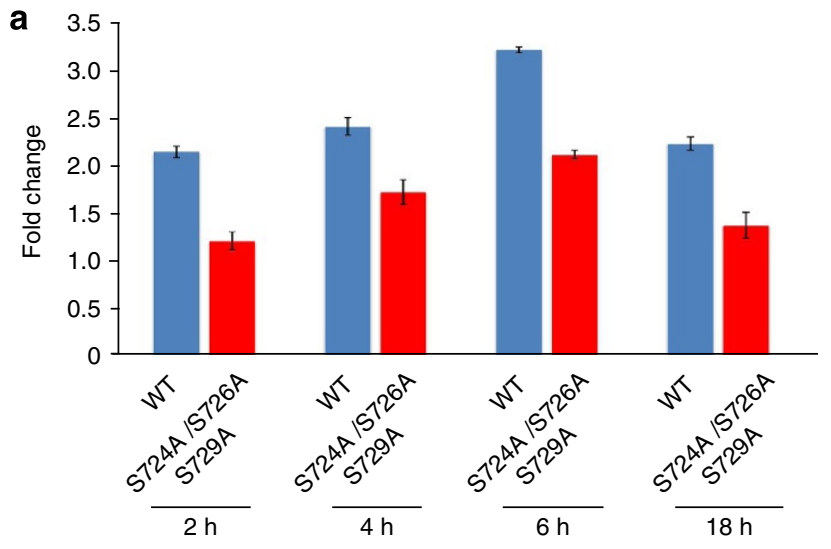

b
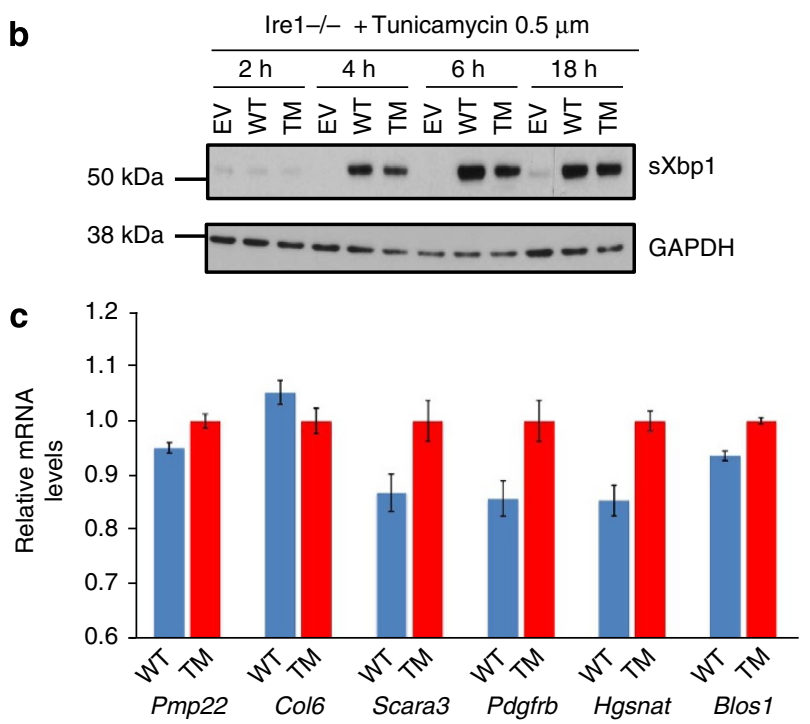

Figure 5 | Activation loop phosphorylation mutants retard splicing and RIDD activity in Ire1 - / - cells. (a) Ire1 - / - cells were co-transfected with plasmids containing Ire1 wild-type (WT), triple activation mutant (TM) or empty vector with plasmid containing GFP. Cells were treated with mild ER stress $(0.5 \mu \mathrm{M}$ tunicamycin), and splicing levels (sXbp1) were measured at 2, 4, 6 and $18 \mathrm{~h}$ time points by qRT-PCR analysis (mean \pm s.e.m., $n=3$ ). The fold change in splicing levels of wild-type and triple mutant Ire1 were compared with empty vector splicing. (b) Western blot using an anti-sXbp1 antibody showing spliced $\mathrm{Xbp} 1$ protein expression levels under mild ER stress at 2, 4, 6, $18 \mathrm{~h}$ time points. Triple mutant shows reduced spliced Xbp1 protein expression levels as compared with wild type in Ire1-/ - cells, which become less pronounced over time. (c) Ire1 - / - cells were treated with $0.5 \mu \mathrm{M}$ tunicamycin for $6 \mathrm{~h}$, after which mRNA levels of RIDD target genes were measured by qRT-PCR and normalized to GAPDH (mean \pm s.e.m., $n=3$ ). mRNA levels of RIDD target genes were lower in cells transfected with WT Ire1 relative to activation loop mutant Ire1.

splicing data. Furthermore, fluctuations in wild-type splicing suggest that wild-type Ire1 may be able to adapt its splicing requirements better than mutant protein, again suggesting that active site phophorylations have a role in regulating splicing output. To investigate the effects of activation loop phosphorylation mutant upon expressed protein levels, we performed a western blot analysis for spliced Xbp1 from Ire1-/ - cells at varying time points. (Fig. 5b, and Supplementary Fig. 6). We clearly see a difference between wild-type and mutant samples for spliced Xbp1 protein expression levels at all time points tested using mild ER stress, with the largest difference in protein levels occurring at a 4-h time point. The difference in spliced Xbp1 expression levels between triple mutant and wild type becomes much less pronounced during the course of the experiment, suggesting that the rate of activation is compromised more severely in the mutant protein during early stages of ER stress.

Taken together, the experiments conducted both in HT1080 and Ire $1-I-$ cells reinforce the notion that activation loop phosphorylations are coupled to RNase activity as evidenced by both reduced mRNA splicing and reduced sXbp1 protein expression levels.

Activation loop phosphorylation mutant reduces RIDD activity. To understand whether active site phosphorylations have an impact upon RIDD, we measured the relative mRNA levels of RIDD target genes ${ }^{21}$ in Ire $1-/$ - cells that have been transfected either with wild-type Ire1 or with triple activation loop mutants using qRT-PCR. After $6 \mathrm{~h}$ of treatment with tunicamycin, we observed that five out of the six RIDD genes had lower mRNA levels in wild-type Ire1 samples as compared with mutant samples with Scara3, Pdgfrb and Hgsnat displaying almost 20\% reduction in wild type compared with mutant (Fig. 5c). This suggests that RIDD activity is higher in wild-type than in mutant samples consistent with the notion that Irel active site phosphorylation mutants reduce RNase activity. Thus, mutation of Irel activation loop phosphorylations reduces Irel's RIDD activity and suggests that phosphorylation may have a regulatory role in RIDD activity.

\section{Discussion}

In this study, we identify specific phosphorylations upon Ire1 that fall into three regions; linker, activation loop and RNase domain. We show that activation loop phosphorylations are important for achieving high levels of splicing in vitro and that mutation of activation loop phosphorylations retards splicing both in HT1080 and in Ire $1-/-$ cells. Moreover, we show that these activation loop phosphorylations have an effect on Irel's RIDD activity. Thus, the data clearly link the phosphorylation events on ser724, ser726 and ser729 within the activation loop to enhanced RNase splicing and imply that kinase activation loop phosphorylations are an important and necessary step to achieve the activated Ire1 RNase state leading to enhanced Xbp1 splicing and subsequent UPR activation.

The precise mechanism of how phosphorylations activate the RNA splicing inferred from this study, and in analogy to other kinases $^{22}$, is most likely based on conformational changes that take place within the protein. In general kinase biology 22 , the phosphorylation event causes movements within the active site that are transmitted to the rest of the protein, manifesting in gross movements of C-lobe relative to N-lobe. In a similar manner, it is most likely that Ire1 activation loop phosphorylation leads to subtle movements within the kinase active site that are transmitted to the RNase active site by conformational movements via the C-lobe of the kinase domain. These conformational changes would induce the binding of RNA substrate in a more efficient manner leading to increased splicing.

Interestingly, we see effects on splicing activity that are consistent between both in vitro and in vivo systems; however, the scale varies. This is most likely due to different rate limiting steps. The rate limiting step in vitro may be the conformational changes relating to association and dissociation of the RNA substrate, whereas in vivo the RNase domain may require other protein or cellular factors to regulate such events. This may cause differences between observations in vitro and in vivo, and such effects cannot be ruled out in this study.

To obtain the differently phosphorylated Ire1 protein for our in vitro analysis, we have made use of eukaryotic insect expression 
system that can authentically process post-translational modifications, in part, due to the presence of phosphatases, and that allows us to isolate distinct phosphorylated species. The ability to purify these distinct populations suggests that they are in a biologically relevant state. Interestingly, we see no further phosphorylation species above those seen in PK4 and no evidence of hyperphosphorylation similar to that seen in yeast Ire 1 expressed in E.coli. A likely explanation for this is that yeast Ire1 has an extra loop insertion rich in ser and thr residues compared with the human sequence, which could be the site for extra hyperphosphorylations; alternatively, a recent report suggests a non-physiological role for hyperphosphorylation for proteins expressed in E.coli $^{23}$.

Although the role of activation loop phosphorylations ser724, ser726 and ser729 is clearly linked to enhanced splicing, the remaining phosphorylations, notably the linker region (L-phos) and RNase domain thr973, have minimal impact on splicing. In the case of L-phos, it is too far way from the kinase or RNase active site to cause an effect. It has be suggested that maybe L-phos has a role in increasing the oligomeric state of Ire1; however, we see no biophysical evidence that any phosphorylated species exists higher than that of a dimer (Prischi et al. in preparation), and even if it did increase the oligomeric state we see no increase in enzymatic rates. Similarly, L-phos could cause the break-up of a dimer, but again this is unlikely as we see no corresponding loss in enzymatic rate when compared with dephosphorylated protein. Interestingly, when we allow the autotransphosphorylation reaction to occur with dephosphorylated protein in vitro, we only measure ser724 and ser726 phosphorylation. The mechanism of activation loop phosphorylation whether in vitro or in cells can easily be rationalized by the human Ire1 crystal structure, which captures the autophosphorylation event and shows the ordered region of the activation loop protruding outward towards the kinase active site of the opposite monomer. However, there must be some cellular factor causing the phosphorylation of ser729, linker region and thr973, possibly another phosphorylating kinase. For ser729, the phosphorylation of which causes an increase in splicing, this could act as another level of regulatory control whereby initial cleavage of Xbp1 causes a tempered response to unfolded protein, but then Ire1 has another 'gear' so as to amplify the response. Very low levels of thr973 phosphorylation were detected and only when the Ire1 protein had already achieved high degree of phosphorylation (PK3, PK4). thr973 does not have an impact directly on the splicing activity in vitro, but has a small noticeable effect in vivo. Its position within the RNase binding domain suggests that it may be able to influence the reaction in other ways. It is tempting to speculate that this phosphorylation and the phophorylations on the linker region could act as recruitment factors for Traf2 binding and initiate apoptosis (Fig. 6a,b). Interestingly, there is now accumulating evidence to suggest that there are a number of factors that can influence the duration and amplitude of Ire1 signalling via a protein-signalling platform termed UPRosome $e^{24,25}$. One such example is the regulation by the BAX-BAK protein complex, which influences Irel signalling by helping to alleviate stress in the first instance, but if the stress signal is not relived then this complex switches to outputting an apoptotic response ${ }^{24}$. It may be that phosphorylations are a key

a

In vitro dephosphorylated Ire1, re-phosphorylated with ATP

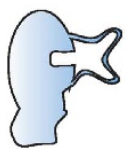

Dephosphorylated Ire1 'ground state'

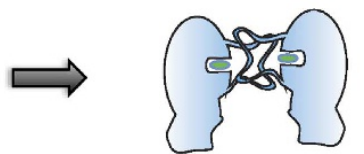

Trans-autophosphorylation complex-bound ATP
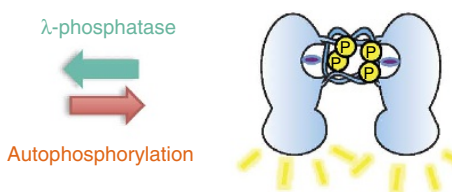

Activation loop phosphorylation ser724 ser726 activates RNase

b

Human Ire1 (expressed in insect cells) phosphorylation model m
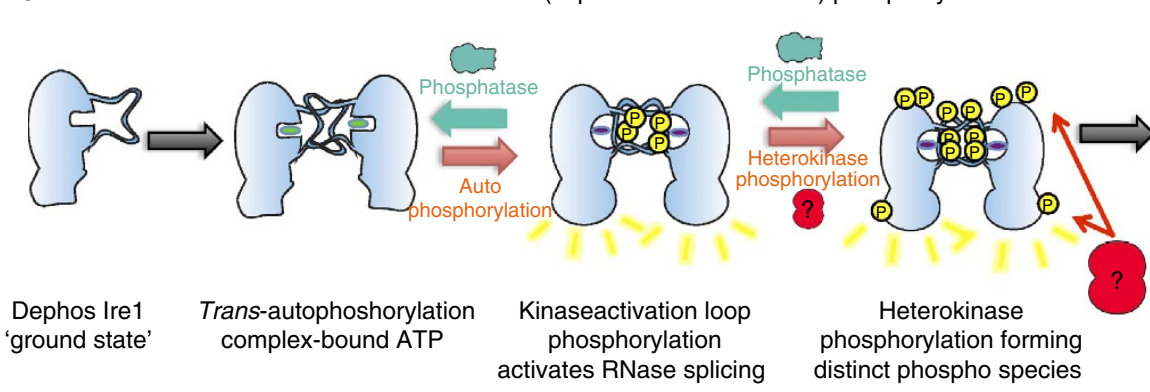

Heterokinase phosphorylation forming distinct phospho species

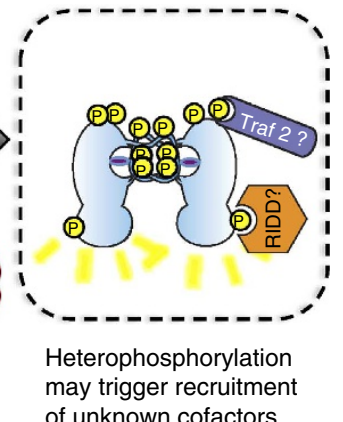

of unknown cofactors

Figure 6 | Model of phosphoregulated Ire1 activation. (a) In vitro dephosphorylated Ire1, re-phoshorylated with ATP. Depicts the situation when purified dephosphorylated human Ire1 protein (PK1) is auto re-phosphorylated in vitro with incubation of ATP only. Initially, the dephosphorylated protein exists in a ground state and forms a face-to-face dimer. Upon addition of ATP, trans-phosphorylation occurs on positions ser724 and ser726 of the activation loop, which causes conformation changes within the kinase domain that translate to the RNase domain resulting in activation of RNase such that splicing turnover is enhanced over 60 -fold higher than that of the 'ground state'. Ire1 then probably reorientates to form a back-to-back arrangement. (b) Recombinant human Ire1 expressed in insect cell model. Initially, Ire1 is dephosphorylated and in the 'ground state' where it forms the transautophosphorylation face-to-face dimer. Upon addition of ATP, autophosphorylation occurs on the activation loop resulting in pSer724, pSer726. Another factor may cause phosphorylation at other sites within the linker region and RNase domain. Phosphatases within eukaryotic cells keep phosphorylated Ire1 in a biologically relevant state. The order of phosphorylation is not known, but the final phosphorylation on the activation loop pSer729 results in activated RNase splicing such that the turnover is enhanced at least 100-fold above ground state. Phosphorylations within the RNase domain and linker region have no/little direct impact on splicing but may be recruiting factors for other process such as RIDD or Traf2; this part of the model is highlighted with dashed lines to indicate that it is speculative. At this stage, Ire1 probably reorientates to form a back-to-back arrangement. 
component of initiating interactions with cofactors that constitute the UPRosome, and hence the importance of the present study is in trying to shed light on this interesting type of regulation.

It is clear that phosphorylation has an important role in regulating Irel activity and what is understood from this study is the impact that specific phosphorylations have on RNase splicing and RIDD activity and the requirement of kinase loop phosphorylations to achieve the activated Ire1 RNase splicing state for UPR signal activation.

\section{Methods \\ Expression and purification of phosphorylated Ire1 species. The C-terminal domain of human Ire1 $\alpha$ (residues 547-977) was expressed in sf9 insect cells using the Bac-to-Bac method after a 3-day-growth at $27^{\circ} \mathrm{C}$ (ref. 3). The cell pellet was lysed by sonication in $50 \mathrm{mM}$ Hepes, $\mathrm{pH} 7.5,300 \mathrm{mM} \mathrm{NaCl}, 10 \%$ glycerol, supplemented with protease inhibitors and centrifuged to remove cell debris and insoluble material at $16,000 \mathrm{~g}$ for $60 \mathrm{~min}$. The supernatant was then passed through a batch/gravity column containing $15 \mathrm{ml}$ of talon resin (Clontech), and protein was eluted off with $300 \mathrm{mM}$ imidazole in a total volume of $50 \mathrm{ml}^{3}$. Sample was treated with Rhinovirus 3C protease (PreScission protease, Amersham Biosciences, $300 \mathrm{ml}$ at $2 \mathrm{mg} \mathrm{ml}^{-1}$ ) to remove his-tag. To obtain distinct Irel phosphorylated species, the lambda phosphatase incubation step was omitted ${ }^{3}$ and sample was passed through Mono-Q column with a shallower gradient $(80-300 \mathrm{mM} \mathrm{NaCl}$ over 30 column volumes). The eluted phosphorylated peaks were separated and further purified by size exclusion chromatography on a Superdex 200 column (GE Healthcare) in a $25 \mathrm{mM}$ Hepes pH 7.5 buffer, containing $150 \mathrm{mM} \mathrm{NaCl}, 5 \mathrm{mM}$ DTT and $5 \%$ glycerol.}

In vitro autophosphorylation. Ire1 autophosphorylation was achieved by incubation for at least $2 \mathrm{~h}$ of $50 \mu \mathrm{M}$ PK1/dephosphorylated Irel with $2 \mathrm{mM}$ ATP (Sigma), $5 \mathrm{mM} \mathrm{MgCl}_{2}$, at room temperature in a $25 \mathrm{mM}$ HEPES, $\mathrm{pH} 7.5$, containing $150 \mathrm{mM} \mathrm{NaCl}, 5 \mathrm{mM}$ DTT and $5 \%$ glycerol. The presence of autophosphorylation was confirmed by mass spectrometry and western blot using a pSer724 phospho-specific antibody.

In vitro RNase splicing assay. Ire $1 \alpha$ RNase activity was measured by incubation of $0.2 \mu \mathrm{M}$ purified protein with increasing concentrations $(0-50 \mu \mathrm{M})$ of quenched single-strand RNA probe ( $5^{\prime}$ - Cy3-GACGUCCACAUCCUGGUCC-BHQ2 - $3^{\prime}$ IDT DNA Technologies, Leuven, Belgium) at $30^{\circ} \mathrm{C}$ in a 384 -well low-volume nonbinding plate (Greiner Bio-One Ltd, Gloucestershire, UK) with a final reaction volume of $20 \mu \mathrm{l}$. Ire $1 \alpha$ reaction buffer $(25 \mathrm{mM}$ HEPES pH 7.5, $150 \mathrm{mM} \mathrm{NaCl}$, $5 \mathrm{mM}$ DTT, $5 \%$ glycerol) was used for all RNase activity measurements. Whether in presence of ATP or ADP or AMP-PNP (Sigma) the same protocol of the autophosphorylation was used (incubation of $50 \mu \mathrm{M}$ protein for at least $2 \mathrm{~h}$ with $2 \mathrm{mM}$ nucleotide and $5 \mathrm{mM} \mathrm{MgCl}_{2}$ ). Time-dependent fluorescence was measured on a POLARstar Omega plate reader (BMG LABTECH GmbH, Germany) using an excitation filter of $550 \mathrm{~nm}$ and emission filter of $590 \mathrm{~nm}$. Initial rates were measured using MARS Data Analysis Software V2.41 (BMG LABTECH GmbH, Germany). Initial rates were plotted with a Michaelis and Menten model using PRISM 6 (GraphPad Software, La Jolla California USA, www.graphpad.com).

Cell culture and in vivo Xbp1 mRNA splicing assays. Human Fibrosarcoma HT1080 cells were cultured in Dulbecco's Modified Eagle Medium supplemented with $10 \%$ Fetal Bovine Serum, $2 \mathrm{mM}$ L-Glutamine, 50U Penicillin per $50 \mu \mathrm{g}$ Streptomycin per $\mathrm{ml}$. A day before transfection, 300,000 cells per well $(2 \mathrm{ml})$ were plated on a six-well plate. Wells were transfected with $2 \mu \mathrm{g}$ of DNA mixed with Fugene 6 reagent (Promega) in a 1:3ratio. $2 \mu \mathrm{g}$ of total DNA per well were prepared by mixing equimolar ratio (1:1) of Wild-Type/Mutants IRE1 $\alpha$ (Adgene pcDNA3 plasmid) and Adgene's pEGFP-C3 (transfection efficiency indicator). After $24 \mathrm{~h}$, IRE1 $\alpha$ - expressing cells in each well were induced by $0.5 \mu \mathrm{M}$ Tunicamycin dissolved in DMSO $(0.5 \% \mathrm{v} / \mathrm{v})$ and harvested after 2,4 and $6 \mathrm{~h}$. The total RNA was extracted from the cells by using RNeasy Plus Mini Kit (Qiagen), and were DNAse I treated (1U/10 $\mu \mathrm{l}$ RNA) and reverse transcribed to cDNA by SuperScript III FirstStrand Synthesis SuperMix (by random hexamers). The spliced (228 bp) and unspliced (254 bp) Xbp1 cDNA fragments were amplified by PCR (Phusion Flash High Fidelity PCR Master Mix-Thermo Scientific) using $68^{\circ} \mathrm{C}$ as an annealing temperature and following set of primers: foward primer: $5^{\prime}$-CCTGGTTGCTGAA GAGGAGG-3' and reverse primer: $5^{\prime}$-CCATGGGGAGATGTTCTGGAG- $3^{\prime}$. PCR products were run on $3 \%$ agarose gel stained with Gel Red dye (Biotium) in $1 \times$ TBE buffer. All samples were made in triplicate and subjected to densitometry measurements by Phoretix gel analysis software (TotalLab). Splicing percentage values are shown as an average value of three measurements \pm s.d. The same experiments were performed in Ire $1-/$ - cells. Ire $1-/-$ MEFs (kindly provided by Professor David Ron) were grown in Dulbecco's Modified Eagle Medium (high glucose) supplemented with $10 \%$ Fetal Bovine Serum, $2 \mathrm{mM}$ L-glutamine, $1 \times$ nonessential amino-acid solution, $50 \mu \mathrm{M}$ 2-Mercaptoethanol and 50U Penicillin per $50 \mu \mathrm{g}$ Streptomycin per ml. A total of 500,000 cells were plated $24 \mathrm{~h}$ before transfection, then transfected with $2 \mu \mathrm{g}$ DNA (equimolar mixture of plasmids + pEGFP-C3) in ratio 1:6 with Fugene 6 reagent (Promega). After $48 \mathrm{~h}$ (peak of IRE1a expression), cells were induced with $5 \mu \mathrm{M}$ tunicamycin and harvested, respectively, after 2, 4 and $6 \mathrm{~h}$. Further stages of experiment were performed as described previously.

Quantitative RT-PCR. MEF Ire 1 - / - retrotranscribed cDNA was used in triplicate for quantitative real-time PCR analysis using the SYBR Green reagent system (Applied Biosystems) and an ABI 7500 (Applied Biosystems). Relative quantities of amplified cDNAs were then determined using SDS v 1.4 software and normalized to GAPDH.

Western blot analysis. Induced MEFs in each well of asix-well plate containing IRE1a - / - cell monolayer were lysed in RIPA buffer, scraped and agitated for $15 \mathrm{~min}$ at $4^{\circ} \mathrm{C}$. After that cell extracts were incubated on ice for $30 \mathrm{~min}$ and centrifuged for $10 \mathrm{~min}$ at $10,000 \mathrm{~g}$. Collected supernatants were mixed with Leammli buffer and run on $4-12 \%$ precast Bis-Tris gels (Invitrogen). Gels were transferred to nitrocellulose membrane (Invitrogen's iBlot) and blocked in PBS $+0.05 \%$ Tween-20 and 5\% Marvel Dried Milk. Primary antibody was added to blocking buffer (PBS, Tween-20, 2.5\% milk powder) in concentration of 1:200 for antispliced human Xbpls (Biolegend) and 1:2,000 for antihuman GAPDH FL-335 (Santa Cruz Biotechnology)(Full image western blot of sXbp1 over time in Ire1 $-/$ - cells is presented in Supplementary Fig. 7). After a $1 \mathrm{~h}$ incubation at room temperature, membranes were washed three times in PBS $+0.05 \%$ Tween-20 and incubated with secondary antibody (anti rabbit, GE Healthcare) diluted $(1: 2,000)$ in $2.5 \%$ milk blocking buffer ( $1 \mathrm{~h}$, room temperature). Followed by another three washes, blots were visualized by Millipore Luminata Crescendo Western HRP substrate and developed on Amersham Hyperfilm ECL (GE Healthcare).

Statistical methods. All experiments were performed in triplicate unless otherwise stated.

\section{Mass spectrometry.}

MS MALDI TOF. Mass spectrometry. In preparation for mass spectrometry, the desired protein band was excised, lyophilized and digested with trypsin (E.C.3.4.21.4, Promega) overnight. Peptides were extracted from gel pieces, and nanoLC was performed on an Ultimate 3000 using a PepMap $10075 \mathrm{~mm} \times 15-\mathrm{cm}$ fused silica C18 analytical column (LC Packings, Dionex, Sunnyvale, CA), coupled to a Probot for fraction collection and matrix addition with $\alpha$-Cyano-4-hydroxycinnamic acid acid as the matrix. A gradient of $2-60 \%$ ACN in $0.1 \%$ TFA was delivered over $36 \mathrm{~min}$ at a flow rate of $0.300 \mathrm{nl} \mathrm{min}^{-1}$. MALDI TOF/TOF-MS was performed using an Applied Biosystems 4800 mass spectrometer (Foster City, CA.) in the positive reflectron mode with delayed extraction. MS precursor acquisition was followed by interpretation and data-dependent MS/MS acquisition with the CID on. Data interpretation was configured to select a maximum of 10 precursor ions per fraction with a minimum signal-to-noise ratio of 50 . The data were processed using GPS Explorer (Applied Biosystems, CA) against the Swiss-Prot database. Search parameters were enzyme $=$ trypsin; fixed modifications $=$ carboxymethyl $(\mathrm{C})$; variable modifications $=$ oxidation $(\mathrm{M})$; mass tolerance \pm 100 p.p.m.; fragment mass tolerance $=0.3 \mathrm{Da}$; maximum missed cleavages $=1$; mass values $=$ monoisotopic. Phosphorlyated peptides were assigned manually on inspection of the data.

Mass analysis of intact protein. Samples were desalted by adsorption to Millipore C4 Ziptip, washed with 5\%(v/v) acetic acid and eluted to the sample slide with $2 \mu \mathrm{l}$ of $\mathrm{CHCA}$ matrix ( $10 \mathrm{mg} \mathrm{ml}^{-1}$ alphacyano-4-hydroxycinnamic acid in $50 \%$ acetonitrile/water conatining $0.1 \%$ trifluroacetic acid). After drying at room temperature, samples were analysed in a Waters MaldiMicro MX mass spectrometer operating in linear mode with a $10 \mathrm{~Hz}$ UV laser, power 130 , pulse voltage $850 \mathrm{~V}$, ion extraction delay time 2,000 ns with external calibration using multiple charge states of bovine trypsinogen and its dimer. Spectra were displayed as the average of $\sim 1,000$ laser shots each. Data processing was performed by using Waters MassLynx software.

ESI QTOF. Sample processing. For intact molecular mass determination, samples were button dialysed (Hapton Research, Aliso Viejo, CA, USA) overnight into $50 \mathrm{mM}$ ammonium acetate, $\mathrm{pH}$ 6.9. For phosphorylation site analysis, samples were separated by 1D SDS-PAGE. The phosphoprotein band was excised for proteolytic digestion with trypsin followed by phosphopeptide enrichment by $\mathrm{TiO}_{2}$ chromatography. In brief, $2.5 \mu \mathrm{g}$ of each protein was mixed $1: 1: 1(\mathrm{v} / \mathrm{v} / \mathrm{v})$ with $3 \times$ Tris sample buffer and $150 \mathrm{mM}$ DTT and heated at $95^{\circ} \mathrm{C}$ for $5 \mathrm{~min}$. Samples were cooled, centrifuged and loaded onto $15 \%$ Tris-glycine gels. A constant voltage of $200 \mathrm{~V}$ was applied for $1.5 \mathrm{~h}$ (running buffer $1 \times$ SDS tris/glycine). The gels were rinsed with distilled water $(5 \mathrm{~min})$ and then stained with InstantBlue Coomassie stain (Expedeon Hartson, UK) overnight. Phopshoprotein gel bands were excised and chopped into small pieces $\left(\sim 1 \mathrm{~mm}^{3}\right)$ and destained in $30 \%$ ethanol at $70{ }^{\circ} \mathrm{C}$ for $30 \mathrm{~min}$ with shaking. This was repeated with fresh ethanol solution until all coomassie stain was removed. The gel was then covered with $25 \mathrm{mM}$ ammonium 
bicarbonate $/ 50 \%$ acetonitrile and vortexed for $10 \mathrm{~min}$. The gel slices were then covered with $100 \%$ acetonitrile and left for $5 \mathrm{~min}$ with vortexing before the supernatant was discarded and replaced with a fresh aliquot of acetonitrile. Acetonitrile was removed, and the gel pieces were completely dried under vacuum centrifugation for $30 \mathrm{~min}$. Once dry, the gel slices were cooled on ice and then covered with ice cold trypsin solution $\left(20 \mathrm{ng} \mathrm{hl}^{-1}\right.$ in $25 \mathrm{mM}$ ammonium bicarbonate) and left on ice for $30 \mathrm{~min}$ to rehydrate. Excess trypsin solution was removed, and the gel slices were covered with a minimal amount of $25 \mathrm{mM}$ ammonium bicarbonate. After briefly vortexing and centrifuging, the gel slices were incubated at $37^{\circ} \mathrm{C}$ with shaking for $18 \mathrm{~h}$. The resulting digest was vortexed, centrifuged and $50 \mu \mathrm{l}$ water was added. Following vortexing for $10 \mathrm{~min}$, the supernatant was recovered and added to an eppendorf containing $5 \mu \mathrm{l}$ acetonitrile/ water/formic acid (60/35/5; v/v). Fifty microlitres of acetonitrile/water/formic acid $(60 / 35 / 5 ; \mathrm{v} / \mathrm{v})$ was added to the gel slices and vortexed for an additional $10 \mathrm{~min}$. The supernatant was pooled with the previous wash, and one additional wash of the gel slices was performed. The pooled washes containing the peptides were dried by vacuum centrifugation. For phosphopeptide enrichment by $\mathrm{TiO}_{2}$ affinity chromatography, the tryptic digests were reconstituted in $25 \mu$ loading buffer $(1 \mathrm{M}$ glycolic acid (Sigma Aldrich, UK) in $80 \%$ acetonitrile/5\% TFA). A $\mathrm{TiO}_{2}$ microcolumn was prepared by plugging the constricted end of a $200 \mu$ l GELoader pipette tip with a $\mathrm{C} 8$ disc. $\mathrm{TiO}_{2}$ beads (GL Sciences, Japan) were suspended in $100 \%$ acetonitrile and packed to column length of $3 \mathrm{~mm}$ by the application of gentle air pressure. The column was flushed with $50 \mu \mathrm{l}$ of $50 \%$ acetonitrile and equilibrated with $20 \mu \mathrm{l}$ of loading buffer. The peptide solution was then passed through the column and the flow-through collected. The column was washed with $5 \mu \mathrm{l}$ of loading buffer followed by $30 \mu \mathrm{l}$ of washing buffer ( $80 \%$ acetonitrile/1\% TFA) ,and the eluates were pooled with the flow-through. Bound peptides were eluted from the column with $50 \mu \mathrm{l}$ elution buffer $(0.5 \%$ ammonia solution) followed by $1 \mu \mathrm{l}$ of $30 \%$ acetonitrile. All fractions were lyophilized to complete dryness and stored at $-20{ }^{\circ} \mathrm{C}$ until ready for MS analysis.

Enriched samples were reconstituted in $10 \mu \mathrm{l}$ water and desalted using ZipTip C18 tips (Millipore UK Ltd, Watford, UK) into 50\% acetonitrile and analysed by Z-spray nanoelectrospray ionization MS using a quadrupole-IMS-orthogonal timeof-flight MS (Synapt HDMS, Waters UK Ltd., Manchester, UK) using gold/ palladium-coated nanospray tips. The MS was operated in positive TOF mode using a capillary voltage of $1.5 \mathrm{kV}$, cone voltage of $20 \mathrm{~V}$, nanoelectrospray nitrogen gas pressure of $0.1 \mathrm{bar}$, backing pressure of $2.47 \mathrm{mbar}$ and collision energy of $15-25 \mathrm{~V}$ in the trap. The source and desolvation temperatures were set at 80 and $150^{\circ} \mathrm{C}$, respectively. During TOF-MS acquisition, Argon was used as the buffer gas, at a pressure of $4.0 \times 10^{-3} \mathrm{mbar}$ in the trap and transfer. Mass calibration was performed by a separate injection of sodium iodide at a concentration of $2 \mu \mathrm{g} \mu \mathrm{l}^{-1}$. Data processing was performed using the MassLynx v4.1 suite of software supplied with the mass spectrometer.

\section{References}

1. Walter, P. \& Ron, D. The unfolded protein response: from stress pathway to homeostatic regulation. Science 334, 1081-1086 (2011).

2. Zhang, K. \& Kaufman, R. J. From endoplasmic-reticulum stress to the inflammatory response. Nature 454, 455-462 (2008).

3. Ali, M. M. et al. Structure of the Irel autophosphorylation complex and implications for the unfolded protein response. EMBO J. 30, 894-905 (2011).

4. Korennykh, A. V. et al. The unfolded protein response signals through highorder assembly of Irel. Nature 457, 687-693 (2008).

5. Lee, K. P. K. et al. Structure of the dual enzyme Irel reveals the basis for catalysis and regulation in nonconventional rna splicing. Cell 132, 89-100 (2008).

6. Yanagitani, K., Kimata, Y., Kadokura, H. \& Kohno, K. Translational pausing ensures membrane targeting and cytoplasmic splicing of XBP1u mRNA. Science 331, 586-589 (2011).

7. Ron, D. \& Walter, P. Signal integration in the endoplasmic reticulum unfolded protein response. Nat. Rev. Mol. Cell. Biol. 8, 519-529 (2007).

8. Hetz, C., Martinon, F., Rodriguez, D. \& Glimcher, L. H. The unfolded protein response: integrating stress singnals through the stress sensor Ire1 $\alpha$. Physiol. Rev. 91, 1219-1243 (2011).

9. Davies, F. E. Insights into the multistep transformation of MGUS to myeloma using microarray expression analysis. Blood 102, 4504-4511 (2003).

10. Carrasco, D. R. et al. The differentiation and stress response factor XBP-1 drives multiple myeloma pathogenesis. Cancer Cell 11, 349-360 (2007).

11. Todd, D. J., Lee, A.-H. \& Glimcher, L. H. The endoplasmic reticulum stress response in immunity and autoimmunity. Nat. Rev. Immunol. 8, 663-674 (2008).

12. Chawla, A., Chakrabarti, S., Ghosh, G. \& Niwa, M. Attenuation of yeast UPR is essential for survival and is mediated by IRE1 kinase. J. Cell Biol. 193, 41-50 (2011).

13. Rubio, C. et al. Homeostatic adaptation to endoplasmic reticulum stress depends on Ire1 kinase activity. J. Cell Biol. 193, 171-184 (2011).
14. Papa, F. R., Zhang, C., Shokat, K. \& Walter, P. Bypassing a kinase activity with an ATP-competitive drug. Science 302, 1533-1537 (2003).

15. Shamu, C. \& Walter, P. Oligomerization and phosphorylation of Irelp kinase during intracelluar signalling from the endoplasmic reticulum to the nucleus. ЕМВO J. 15, 3028-3039 (1996).

16. Tirasophon, W., Welihinda, A. A. \& Kaufman, R. J. A stress response pathway from the endoplasmic reticulum to the nucleus requires a novel bifunctional protein kinase/endoribonuclease (Irelp) in mammalian cells. Genes Dev. 12, 1812-1824 (1998).

17. Jha, B. K. et al. Inhibition of RNase L and RNA-dependent protein kinase (PKR) by sunitinib impairs antiviral innate immunity. J. Biol. Chem. 286, 26319-26326 (2011).

18. Keshwani, M. M., Ross, D. B., Ragan, T. J. \& Harris, T. K. Baculovirusmediated expression, purification, and characterization of a fully activated catalytic kinase domain construct of the $70 \mathrm{kDa} 40 \mathrm{~S}$ ribosomal protein S6 kinase-1 alphaII isoform (S6K1alphaII). Protein Expr. Purif. 58, 32-41 (2008).

19. Klein, S. et al. Expression and purification of active PKB kinase from Escherichia coli. Protein Expr. Purif. 41, 162-169 (2005).

20. Wiseman, R. L. et al. Flavonol activation defines an unanticipated ligandbinding site in the kinase-RNase domain of IRE1. Mol. Cell. 38, 291-304 (2010).

21. Cross, B. C. S. et al. The molecular basis for selective inhibition of unconventional mRNA splicing by an IRE1-binding small molecule. Proc. Natl Acad. Sci. 109, E869-E878 (2012).

22. Huse, M. \& Kuriyan, J. The conformational plasticity of protein kinases. Cell 109, 275-282 (2002).

23. Shrestha, A., Hamilton, G., O’Neill, E., Knapp, S. \& Elkins, J. M. Analysis of conditions affecting auto-phosphorylation of human kinases during expression in bacteria. Protein Expr. Purif. 81, 136-143 (2012).

24. Hetz, C. The unfolded protein response: controlling cell fate decisions under ER stress and beyond. Nat. Rev. Mol. Cell. Biol. 13, 89-102 (2012).

25. Woehlbier, U. \& Hetz, C. Modulating stress responses by the UPRosome: a matter of life and death. Trends. Biochem. Sci. 36, 329-337 (2011).

\section{Acknowledgements}

We thank Professor David Ron for kindly providing us with Irel - / - cells, Professor Xiaodong Zhang and Professor Paul Freemont and their respective labs, particularly Kirsten Jensen, for advice and use of equipment, Dr Ian Collins and Dr Faith Davies for helpful advice and Professor Laurence Pearl for helpful advice and support. We also acknowledge the Centre for Structural Biology. Mass spectrometry was performed at the CISBIO mass spectrometry core facility, managed by Dr Paul Hitchen, at Imperial College London; Dr James Ault at Leeds University and PNAC mass spectrometry facility at Cambridge University. This work was funded from a Cancer Research UK Fellowship (C33269/A11161) awarded to M.A.

\section{Author contributions}

F.P. isolated and purified different phosphorylation species, designed, performed and analysed FRET data, performed and analyzed qRT-PCR data, analysed Mass spec and cloned some in vivo mutants. P.R.N. expressed constructs in sf9 cells, cloned Ire1 mutants, performed and analysed Ire1 in vivo RT-PCR splicing experiment and maintained all cell cultures. M.C. helped with cloning and purification of in vivo mutants. M.M. U.A. conceived, designed and supervised experiments and wrote the manuscript.

\section{Additional information}

Supplementary Information accompanies this paper at http://www.nature.com/nature communications

Competing financial interests: The authors declare no competing financial interests

Reprints and permission information is available online at http://npg.nature.com/ reprintsandpermissions/

How to cite this article: Prischi, F. et al. Phosphoregulation of Ire1 RNase splicing activity. Nat. Commun. 5:3554 doi: 10.1038/ncomms4554 (2014).

This work is licensed under a Creative Commons Attribution 3.0 Unported License. The images or other third party material in this article are included in the article's Creative Commons license, unless indicated otherwise in the credit line; if the material is not included under the Creative Commons license, users will need to obtain permission from the license holder to reproduce the material. To view a copy of this license, visit http://creativecommons.org/licenses/by/3.0/ 


\section{Corrigendum: Phosphoregulation of Ire1 RNase splicing activity}

Filippo Prischi, Piotr R. Nowak, Marta Carrara \& Maruf M.U. Ali

Nature Communications 5:3554 doi: 10.1038/ncomms4554 (2014); Published 7 Apr 2014: Updated 17 Dec 2014

In the Methods section of this Article, an incorrect sequence was provided for the single-strand RNA probe used for in vitro RNA splicing assays. The correct sequence is 5'-Cy3-GAGUCCGCAGCACUC-BHQ2-3'. 